\title{
Primates do not spontaneously use shape properties for object individuation: a competence or a performance problem?
}

\author{
Natacha Mendes $\cdot$ Hannes Rakoczy • \\ Josep Call
}

Received: 15 July 2010/Revised: 4 November 2010/Accepted: 10 December 2010/Published online: 8 January 2011

(C) The Author(s) 2011. This article is published with open access at Springerlink.com

\begin{abstract}
Several recent studies have documented that non-human primates can individuate objects according to property and/or kind information in much the same way as human infants do from around one year of age when they begin to acquire language. Some studies suggest, however, that only some properties are used for the individuation of food items: color, but not shape. The present study investigated whether these findings reveal a true competence problem with shape properties in the food domain or whether they merely reveal a performance problem (e.g., lack of attention to shapes). We tested 25 great apes (chimpanzees, bonobos and gorillas) in two food individuation tasks. We manipulated subjects' experience with differences in color and shape properties of food items. Results indicated (i) that all subjects, regardless of their prior experience, solved the color-based object individuation task and (ii) that only the group with previous experience with different shape properties succeeded in the
\end{abstract}

\footnotetext{
N. Mendes $(\square)$

Institute of Cognitive Neurology and Dementia Research,

Otto-Von-Guericke Universität Magdeburg,

Leipziger Str. 44, 39120 Magdeburg, Germany

e-mail: natacha.mendes@med.ovgu.de

H. Rakoczy

Department of Developmental Psychology Georg-Elias-Müller Institute of Psychology, University of Göttingen, Waldweg 26, 37073 Göttingen, Germany

H. Rakoczy

Courant Research Center "Evolution of Social Behaviour", University of Göttingen, Kellnerweg 6, 37077 Göttingen, Germany

J. Call

Max-Planck-Institute for Evolutionary Anthropology,

Deutscher Platz 6, 04103 Leipzig, Germany
}

shape-based individuation task. Great apes can thus be primed to take shape into account for individuating food objects, and this results clearly speaks in favor of a performance (rather than a competence) problem in using shape for object individuation of food items.

Keywords Object individuation - Shape $\cdot$ Color · Food domain · Primates

\section{Introduction}

Human infants' object cognition has been shown to undergo a developmental shift around the first birthday: while from very early on, infants are capable of tracking objects according to spatiotemporal criteria, only from around 10 to 12 months do they become able to track and individuate objects according to property and/or kind information (e.g., Xu and Carey 1996; see Xu 2007 for a review). As this ability has been found to correlate with natural language comprehension (Xu and Carey 1996) and to reveal itself in linguistically supported contexts specifically (Xu 2002; Xu et al. 2005), one hypothesis is that language is necessary for the development of this very ability (Xu 2002).

Work with non-human primates (hereafter primates), however, puts that bold hypothesis into question. Rhesus monkeys and great apes have been found to individuate objects according to their property/kind much in the same way as human infants from around 1 year old do (Uller et al. 1997; Santos et al. 2002; Phillips and Santos 2007; Mendes et al. 2008): When they see an object with property $\mathrm{X}$ or of kind $\mathrm{A}$ go into an empty box and then find an object with property $\mathrm{Y}$ or of kind $\mathrm{B}$ (unexpected), they search longer than when they find the original object (expected). 
However, mixed results have been found so far concerning the question of which types of properties primates use for individuating objects in different domains. In the domain of food items, the only one which has been used in object individuation studies so far, primates seem to spontaneously use color differences to individuate objects of the same kind, but not shape differences (Santos et al. 2002). That is, when they see, for example, a white food item disappear in an empty box and then find a blue one instead, they continue searching. However, if they see a round food item, they do not respond differently upon finding a triangle one than upon finding the original round one (Santos et al. 2002).

Similar behavior patterns are found in induction tasks in which primate subjects have to decide on the edibility of novel food items (for an overview, see Hauser and Spelke 2004). In one study, for example, an experimenter (E) ate, in full view of rhesus monkeys (Macaca mulatta), a food item which was new to the subjects. E then put down two food items, one identical in color but not in shape to the one $\mathrm{E}$ ate and another one different in color but identical in shape. Monkeys approached significantly more often the food item identical in color but different in shape compared to the item differing in color but identical in shape to the one E ate (Santos et al. 2001; see also Shutts et al. 2009).

This prevalence in the use of color properties over shape properties in object individuation and induction in the food domain stands in contrast to findings from the domain of tool use. Here, primates have been found to rely on shape properties (functionally relevant) and neglect color (functionally irrelevant) when having to choose among tools which are differentially appropriate for a given problem (e.g. Hauser et al. 2002; Santos et al. 2003; Santos et al. 2006; see also Furlong et al. 2008 and Bania et al. 2009 regarding the choice of tools with functional relevant shape properties, over non-functional ones, by chimpanzees).

This raises the question of how the restriction to certain properties, namely color, in primates' individuation of food objects is to be explained, in particular in light of reverse patterns in tool induction tasks. Does this restriction reflect a true competence problem? One way such a competence problem might arise would be that primates' object individuation operates domain specifically and the domainspecific ability is wired such that shape is not a property that enters the picture in the food domain (whereas it does in the domain of tools). Alternatively, the restriction to certain properties found so far might reflect merely some kind of performance problem such that primates can use shape properties to individuate objects but do not spontaneously do so. One possibility along the latter lines would
Table 1 Age, sex, and rearing history of the subjects that participated in the study

\begin{tabular}{|c|c|c|c|c|}
\hline Species & Subject & $\begin{array}{l}\text { Age (years, } \\
\text { months) }\end{array}$ & Sex & Rearing history \\
\hline \multirow[t]{5}{*}{ Bonobo } & Joey & 24.0 & M & Hand reared \\
\hline & Kuno & 10.1 & M & Hand reared \\
\hline & Limbuko & 11.2 & M & Hand reared \\
\hline & Ulindi & 13.2 & $\mathrm{~F}$ & Mother \\
\hline & Yasa & 9.4 & $\mathrm{~F}$ & Mother \\
\hline \multirow[t]{16}{*}{ Chimpanzee } & Alex & 4.9 & M & Hand reared \\
\hline & Alexandra & 6.4 & $\mathrm{~F}$ & Hand reared \\
\hline & Corry & 29.2 & $\mathrm{~F}$ & Hand reared \\
\hline & Dorien & 25.3 & $\mathrm{~F}$ & Hand reared \\
\hline & Fifi & 12.6 & $\mathrm{~F}$ & Mother \\
\hline & Fraukje & 29.10 & $\mathrm{~F}$ & Hand reared \\
\hline & Frodo & 12.2 & M & Mother \\
\hline & Gertrudia & 12.6 & $\mathrm{~F}$ & Mother \\
\hline & Jahaga & 12.11 & $\mathrm{~F}$ & Mother \\
\hline & Lome & 4.3 & M & Mother \\
\hline & Natascha & 25.10 & $\mathrm{~F}$ & Hand reared \\
\hline & Patrick & 8.7 & M & Mother \\
\hline & Pia & 6.4 & $\mathrm{~F}$ & Mother \\
\hline & Riet & 28.3 & $\mathrm{~F}$ & Hand reared \\
\hline & Robert & 30.2 & M & Hand reared \\
\hline & Unyoro & 8.10 & M & Mother \\
\hline \multirow[t]{4}{*}{ Gorilla } & Bebe & 26.7 & $\mathrm{~F}$ & Mother/Hand reared \\
\hline & N'Diki & 28.1 & $\mathrm{~F}$ & Mother/Hand reared \\
\hline & Ruby & 8.0 & $\mathrm{~F}$ & Hand reared \\
\hline & Viringika & 10.9 & $\mathrm{~F}$ & Mother \\
\hline
\end{tabular}

$F$ Female; $M$ Male

be that shape is not salient enough for primates in the food domain, but can be used for object individuation if made more salient.

To test between such competence and performance accounts, two groups of great apes were studied, with the amount of experience with shape and color properties and thus their salience being experimentally manipulated between groups. One group (the "priming group") was primed to attend to three different shapes and colors of food items belonging to the same kind (food pellets). The other group (the "naïve group") remained naïve with regard to shapes and colors other than the "regular" ones (i.e., the regular color (brown) and shape (cylinder shaped) of pellets). Both groups then performed object individuation tasks similar to the ones previously used (Mendes et al. 2008; Santos et al. 2002), and their performance in colorbased and shape-based individuation of food items was compared. 


\section{Methods}

Subjects

Sixteen chimpanzees (Pan troglodytes), five bonobos (Pan paniscus) and four gorillas (Gorilla gorilla) participated in the present study (see Table 1). There were nine males and 16 females. The average age of all the males was 12 years and 4 months, and the average age of all the females was 17 years and 5 months. All subjects were socially housed at the Wolfgang Koehler Primate Research Center, Leipzig Zoo, Germany. Subjects had access to both indoor and outdoor enclosures. All enclosures were furnished with vegetation, climbing structures and visual barriers. Subjects were neither food- nor water-deprived, and they could stop participating in the task at any given moment. All subjects had previously participated in a first study investigating the use of spatiotemporal and property/kind information during an object individuation task (Mendes et al. 2008).

\section{Apparatus}

An opaque plastic box $(40 \times 40 \times 34.5 \mathrm{~cm})$ was used during the experiment (see Fig. 1). The box had a circular opening (approx. $8.5 \mathrm{~cm}$ in diameter) on its top middle part which was used by the experimenter (E) to introduce a food item (pellet). The frontal part of the box (facing the subjects) had an opening (13 cm wide $\times 6 \mathrm{~cm}$ high) which the subjects could use to reach for the pellet. Such opening was covered with a curtain (to avoid subjects looking inside it)

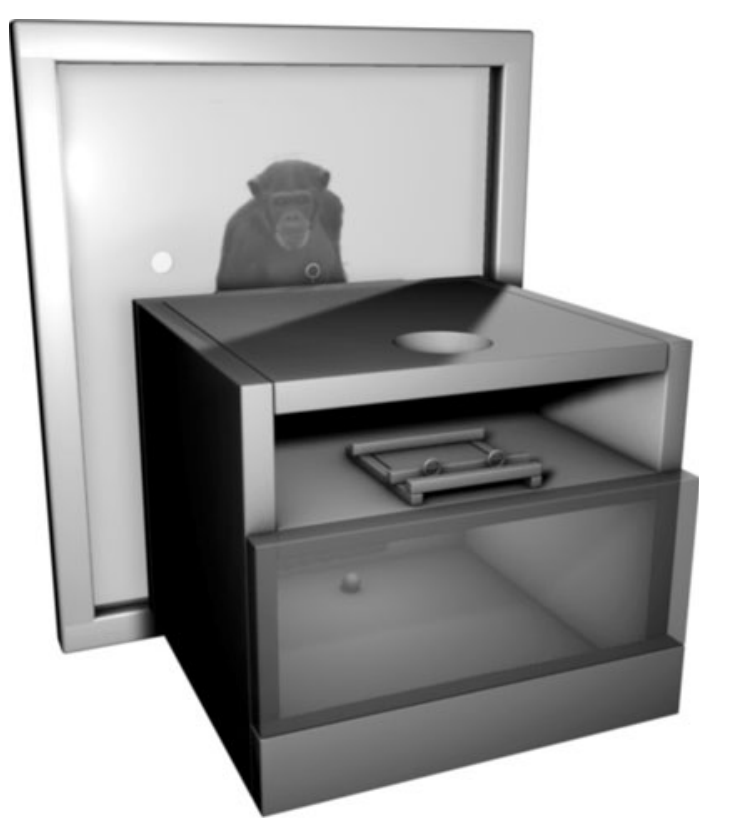

Fig. 1 Drawing of the experimental apparatus (E's perspective). The $\mathrm{X}$-ray view allows the reader to see the location of a pellet inside the box and could be closed using a sliding door manipulated by E. In order to facilitate the surreptitious introduction of the "missing" pellet inside the box (during unexpected trials), a horizontal sliding door was constructed on a false roof of the box ( $9 \mathrm{~cm}$ high from its top part). Subjects were not aware of that. A soft carpet was added to the floor of the box. The carpet prevented the subjects to use possible auditory cues that may have emanated from the fall of the pellets on the floor of the box. The pellets used in the current study were much harder than the food items used in Mendes et al. (2008) and therefore produced a louder sound that this way could be minimized.

In addition to the box, a plastic table $(78 \times 35 \mathrm{~cm})$ was also used but only during the priming phase. The table was attached to a mesh window in the subjects testing room and was used to place different colored or shaped pellets on its top part.

\section{Procedure}

Testing was done by the same $\mathrm{E}$ as in our previous study (Mendes et al. 2008), and the procedure was also very similar to the one used in that study. A cameraperson helped $\mathrm{E}$ by timing the trials and informed $\mathrm{E}$ when the subject had retrieved the pellet from inside the box. The experiment comprised three phases which were always administered in the following order:

\section{Priming phase}

Subjects were divided into two groups. The priming group $(N=13)$ was exposed to three different colors and shapes of pellets. Previous to this study, subjects had never experienced pellets different from the "regular" ones. In contrast, the naïve group $(N=12)$ remained naïve toward colors and shapes other than the regular ones.

During the priming phase, E sat behind a table which was attached to a mesh window in the subjects' room. Subjects received two blocks of trials; one block per each condition (color and shape). The order of presentation of the blocks was counterbalanced across subjects. Each block contained three trials in which the pellets differed within the same property (color or shape). In each trial, the three pellets were placed on the table, aligned in a row and handed one by one to the subject by $\mathrm{E}$. The order of presentation of the pellets on the table was counterbalanced across the three trials of the same block. Each block was presented $24 \mathrm{~h}$ previous to the corresponding testing phase. That is, subjects were exposed to a color priming $24 \mathrm{~h}$ before the testing phase with different colored pellets and analogously for shape priming and the corresponding testing phase. On the day of the testing phase, but previous to its start, the priming group received one more priming 
trial with the same property as the one of the test condition to be next presented.

The colored pellets were red, blue and brownish (i.e., "regular") ones. The red and blue colors were obtained by adding an edible non-flavored food coloring to the "regular" pellets. The shaped pellets had a form of a star, moon and cylinder (i.e. "regular"), all with equal volume. The star and moon-shaped pellets were made from crushed pieces of "regular" pellets, misted with water, molded into the cookie cutters and finally dried.

\section{Familiarization phase}

On the same day, the testing phase began, but immediately before its start, subjects could explore the new the box over a 40-s period. In the case of the priming group, subjects received the familiarization phase immediately after the last priming trial.

\section{Testing phase}

Both priming and naïve groups received two conditions, a color and a shape conditions. Each condition contained four trials, two expected and two unexpected trials. The procedure was identical to the one described previously (Mendes et al. 2008-Experiment 2). In the expected trials, subjects saw $\mathrm{E}$ introducing pellet $\mathrm{A}$ inside the box, and when allowed to reach, they found pellet $A$. In the unexpected trials, the box was initially baited with pellet B (subjects were unaware of this manipulation) and they saw E introducing pellet A. However, pellet A was surreptitiously stored on the horizontal sliding door of the false roof of the box. Once the sliding door was opened, subjects found pellet $\mathrm{B}$, different in properties but not in kind from the one they saw being hidden.

The temporal structure in both expected and unexpected trials was as follows (see Fig. 2 for a schematic illustration of the applied procedure):

(a) Expected trials

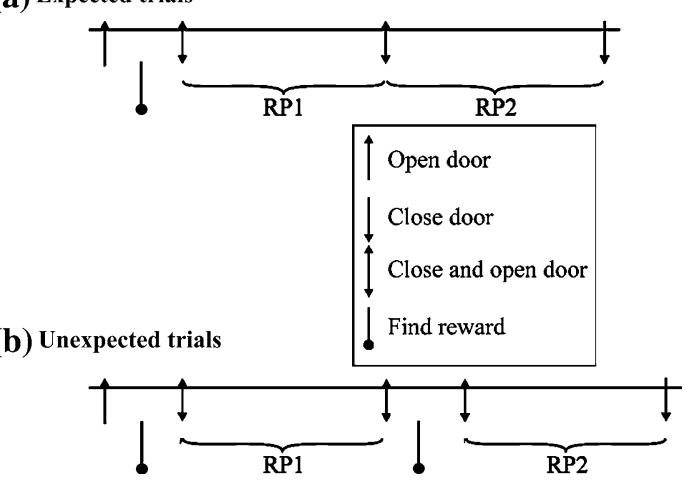

Fig. 2 Illustration of the procedure during expected and unexpected trials
First reach period. Once the subjects had found the pellet, the sliding door of the box was closed immediately for a 20 -s period (RP1).

Intermediate period After the first reach period was over, the sliding door was closed and the horizontal sliding door was simultaneously opened so that the "missing" reward (in the unexpected trials) fell near the front corners of the box. Thus, creating the impression that the reward had always been there and that subjects had not found it before because of its difficult location, not because $\mathrm{E}$ had manipulated it. While E closed and opened the sliding door, simultaneously with the opening of the trap, she spoke loudly (i.e., "Look at that!") to prevent subjects from using auditory cues that may have emanated from the fall of the "missing" pellet (applied in both expected and unexpected trials). If subjects did not reach inside the box or if they failed to find the "missing" pellet in the unexpected trials, the trial was ended after $20 \mathrm{~s}$.

Second reach period If subjects retrieved the "missing" reward, the door was immediately closed and re-opened again for a last 20-s reach period (RP2).

The order of presentation of each condition (i.e., color or shape) was counterbalanced within each group. Within each condition, the order of presentation of the first trial was randomized. Expected and unexpected trials did not occur twice in a row.

\section{Coding and data analysis}

All videos were digitalized and an observer coded them using the Interact ${ }^{\circledR}$ software (version 7). Following the previous study (Mendes et al. 2008), there were two dependent measures: (i) frequency of reaches inside the box and (ii) duration of reaches inside the box. Regarding frequency and duration of reaches, the mean values over the two trials of the same type (expected and unexpected) in each condition (shape and color) were computed. Therefore, each subject had two mean frequency and two mean duration values (corresponding to RP1 and RP2) for expected and unexpected trials in the shape and color conditions. A second observer, blind to the hypotheses of the study, scored a random sample of $20 \%$ of the trials. Inter-observer reliability for frequency was high (Pearson correlation $r=0.985, P<0.001, N=116$ and weighted Kappa $=0.910$ ) as well as for duration of reaches (Pearson correlation $r=0.997, P<0.001, N=116)$.

As the data failed to fulfill the requirement for parametric testing, non-parametric tests were used in all analyses (Kruskal-Wallis, Wilcoxon signed rank test, exact Wilcoxon test for $N \leq 15$ ). We used one-tailed tests because we had clear predictions, i.e., more and longer reaching in unexpected than in expected trials and within unexpected trials more and longer reaching in RP1 than in 
RP2. Regarding the frequency and duration of reaches in RP1 and RP2 of expected trials, no differences were expected.

\section{Results}

Preliminary analyses: species and age effects

First, species and age effect were tested for by using the scores obtained from the difference between (i) expected and unexpected trials within the first reach period (RP1); (ii) expected and unexpected trials within the second reach period (RP2), both for shape and color conditions. No difference in performance between species or age classes (infant: 0-5 year old; juvenile: 5-8 year old; subadult: 8-11 year old; adult $>11$ ) was found for any of the scores aforementioned (Kruskal-Wallis test, all $P>0.110$ ). An exception was a species difference in the shape condition regarding the duration and frequency of reaches both between expected and unexpected trials during the first reach period (RP1) (Kruskal-Wallis test: duration, $H=6.38, P=0.034$; frequency, $H=5.03, P=0.075)$. Because significant $P$ values in each dependent variable might be spurious, Fisher's omnibus test was computed (Haccou and Meelis 1994). The difference in duration and frequency of reaches between expected and unexpected trials during RP1 revealed no significant difference between species (Fisher's omnibus test, duration: $\chi^{2}=8.67, \quad \mathrm{~d} f=6, \quad P=0.19 ; \quad$ frequency: $\quad \chi^{2}=8.02$, $\mathrm{d} f=6, P=0.24)$, thus supporting the view of a spurious significance. Data were, therefore, collapsed across species and age classes.

\section{Main analyses}

The main analyses tested the effect of the priming phase on the mean frequency and duration of reaches during the first and second reach periods (RP1 and RP2).

\section{Shape condition}

If apes individuate object according to shape, two patterns would be expected: (a) in the first reaching period (RP1), subject should search more often and longer in the unexpected than in the expected trials. (b) In the unexpected trials, subjects search longer and more often in RP1 (searching for a missing pellet) than in RP2 (after having found that pellet). Regarding (a), an analysis on the whole sample revealed that during the first reach period (RP1), the priming group reached significantly more often and for longer time during unexpected compared with expected trials (Wilcoxon signed rank test: frequency, $T^{+}=8.5$,
$N=12, P=0.005$; duration, $T^{+}=11, \quad N=12, P=$ 0.013; Fig. 3). In contrast, during RP1, the naïve group did not show any significant differences in frequency or duration of reaching in expected compared with unexpected trials (frequency, $T^{+}=17, N=9, P=0.287$; duration, $T^{+}=25, N=11, P=0.260$; Fig. 3 ).

Regarding (b), the corresponding analyses could only be run with subjects who participated in RP2. In the intermediate period, in each group, not all subjects retrieved the "missing" pellet in at least one of the two unexpected trials $\left(N_{\text {priming group }}=8\right.$ and $\left.N_{\text {naïe group }}=8\right)$. Thus, differences in performance across both reaching periods (RP1 and RP2) was analyzed for this sub-sample only (the same subsample analysis has been previously described elsewhere; see Mendes et al. 2008). During unexpected trials, the priming group reached significantly more often and longer during the first (RP1) compared with the second reach periods (RP2) (frequency: $T^{+}=3, N=8, P=0.023$; duration: $T^{+}=4, N=8, P=0.027$; Fig. 4). However, during the expected trials, the group reached equally often and equally long during RP1 compared with RP2 (frequency: $T^{+}=10, N=7, P=0.625$, two-tailed; duration: $T^{+}=10, N=8, P=0.313$, two-tailed; Fig. 4).

In contrast to the performance of the priming group, during unexpected trials, the naïve group reached equally often during the first (RP1) compared with the second
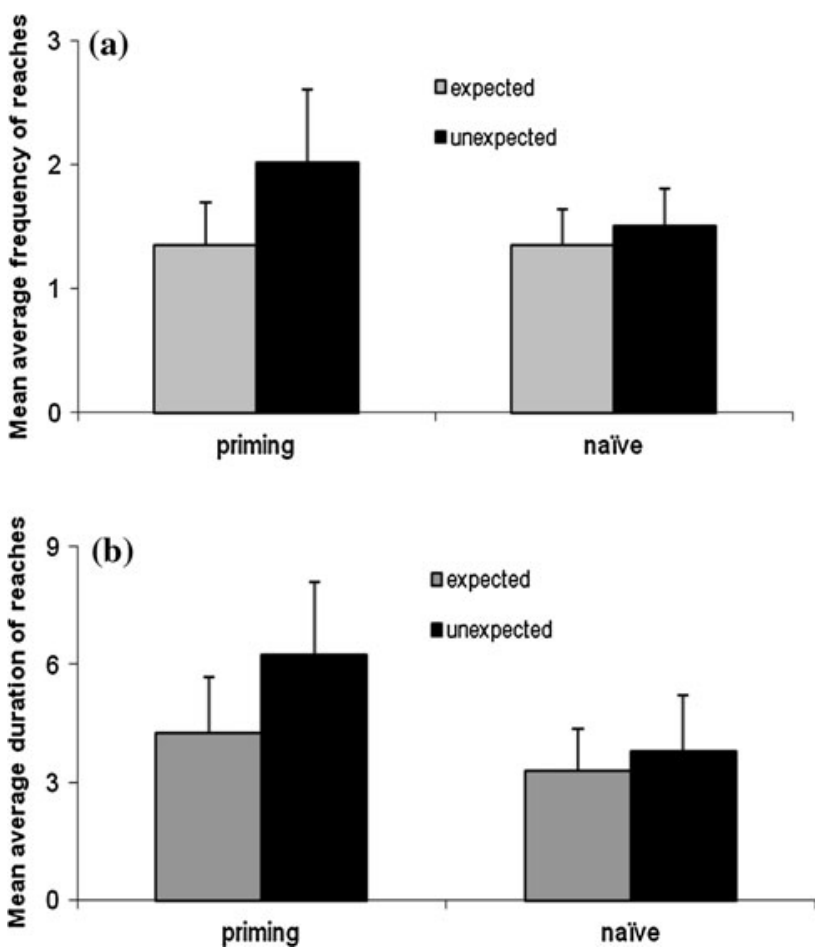

Fig. 3 Mean average (+SE) frequency a and duration $\mathbf{b}$ of reaches during the first 20-s reach period (RP1) in both priming and naïve groups during the shape condition 

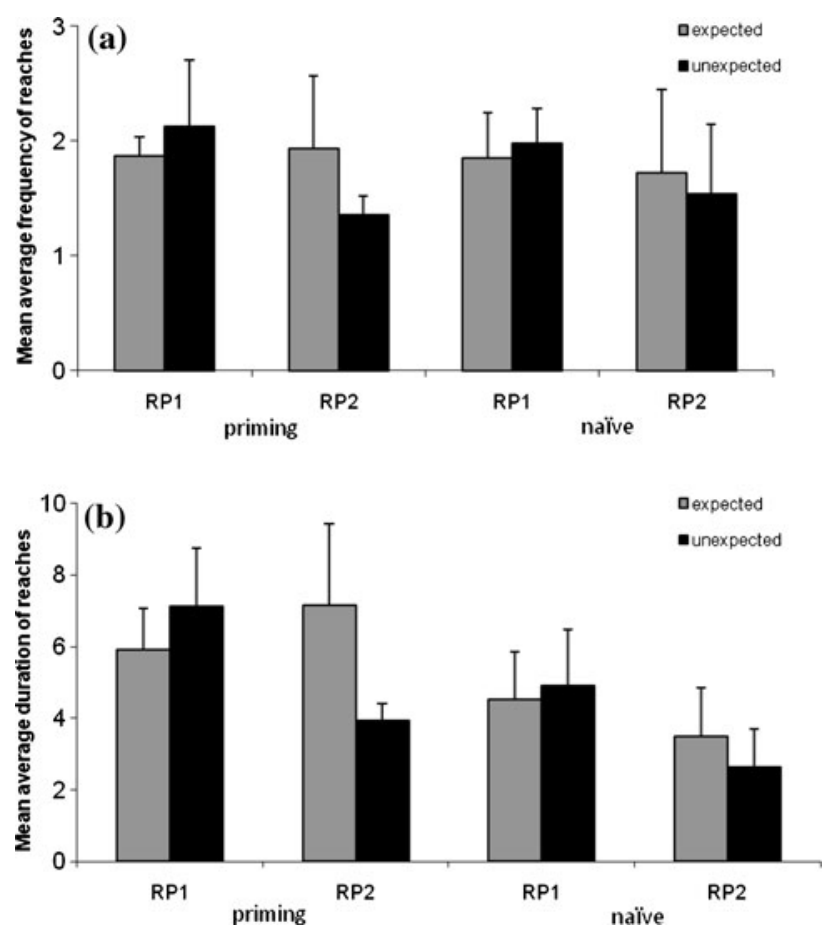

Fig. 4 Mean average (+SE) frequency a and duration $\mathbf{b}$ of reaches during both 20-s reach periods (RP1 and RP2) of those subjects $\left(N_{\text {priming group }}=8\right.$ and $N_{\text {naive group }}=8$ ) who found the "missing" pellet in at least one of the unexpected trials of the shape condition

reach periods (RP2) $\left(T^{+}=8, N=7, P=0.180\right)$. However, the naïve group reached significantly longer during RP1 compared with RP2 $\left(T^{+}=5, N=8, P=0.039\right)$. During expected trials, no significant differences were found between RP1 and RP2 both for frequency and for duration of reaches (frequency: $T^{+}=10, N=6, P=1.0$, two-tailed; duration: $T^{+}=7, N=8, P=0.148$, twotailed; Fig. 4).

\section{Color condition}

In contrast to the performance of the "priming" group in the shape condition during the first reach period (RP1), in the color condition the same group did not show any differences in frequency or duration of reaching in unexpected compared with expected trials (Wilcoxon signed rank test: frequency, $T^{+}=23.5, \quad N=11, P=0.214$; duration, $T^{+}=25, N=12, P=0.151$, Fig. 5). In contrast, during RP1, the "naïve" group reached more often and for longer time during unexpected compared with expected trials (frequency, $T^{+}=10, \quad N=9, \quad P=0.080 ; \quad$ duration, $T^{+}=17, N=11, P=0.087$, Fig. 5).

As for the shape condition, also here only some subjects, from both groups, retrieved the "missing" pellet in at least one of the two unexpected trials $\left(N_{\text {priming group }}=10\right.$ and $N_{\text {naive group }}=8$ ). Thus, as previously conducted, we will
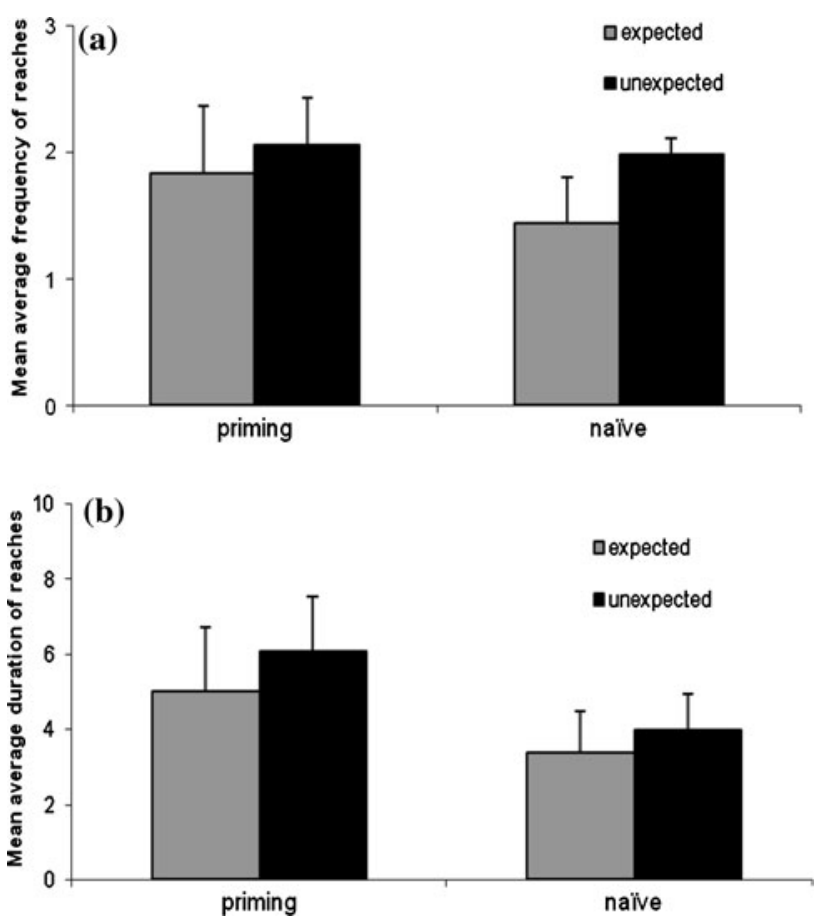

Fig. 5 Mean average ( + SE) frequency a and duration $\mathbf{b}$ of reaches during the first 20-s reach period (RP1) in both priming and naive groups during the color condition

focus on those subjects while analyzing their performances. During unexpected trials, the priming group reached significantly more often and for longer time during RP1 than during RP2 (frequency: $T^{+}=3, N=8, P=0.02$; duration: $T^{+}=9, N=10, P=0.032$, Fig. 6). In contrast, during expected trials, the group reached significantly more often and for longer time during RP2 than during RP1 (frequency: $T^{+}=3, N=8, P=0.031$, two-tailed; duration: $T^{+}=6, N=10, P=0.027$, two-tailed, Fig. 6).

Similar to the performance of the priming group, during unexpected trials, the naïve group also reached significantly more often and for longer time during RP1 compared with RP2 (frequency: $T^{+}=0, N=7, P=0.008$; duration: $T^{+}=4, N=8, P=0.027$, Fig. 6). However, during expected trials, no significant differences were found between RP1 and RP2 both for frequency and for duration of reaches (frequency: $T^{+}=9, N=6, P=0.813$, two-tailed; duration: $T^{+}=9, N=8, P=0.250$, two-tailed, Fig. 6).

\section{Discussion}

Replicating previous work, the present study found that great apes can spontaneously use color properties to individuate objects. The different previous experience (with or without priming) of the two groups did not make much 

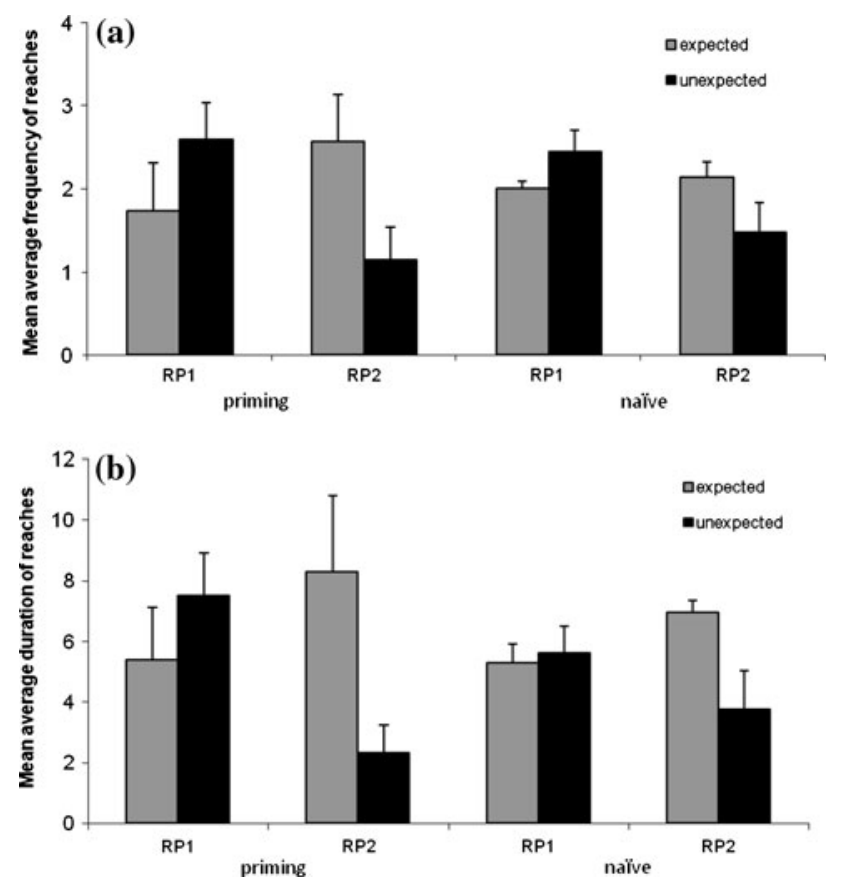

Fig. 6 Mean average (+SE) frequency a and duration $\mathbf{b}$ of reaches during both 20-s reach periods (RP1 and RP2) of those subjects ( $N$ "priming" group $=10$ and $N$ "naive" group $=8$ ) who found the "missing" pellet in at least one of the unexpected trials of the color condition

difference to their use of color: both groups showed increased search behavior when finding a food item of a different color compared to the one they originally saw and showed decrease in search only after finding the original item. With regard to shape properties, in contrast, the present study showed that great apes can use shape to individuate objects, but only after some previous experience: the priming group, unlike the naive group, also showed the above-mentioned search patterns in shapebased individuation tasks. What this clearly suggests is that apes' failure to spontaneously use shape for the individuation of food items that was found in previous studies (and replicated here) does not reflect any deep competence problem. This lack of spontaneous shape-based food object individuation can be alleviated with some previous experience - and with very little and very shallow experience (a couple of encounters with the food items) indeed.

These findings have at least two wider implications. First, there are implications regarding the nature of cognition about food. In comparative and developmental psychology, there is currently some debate about the question of whether and to which degree food constitutes a special cognitive domain with dedicated domain-specific, hard-wired machinery (on a par with naïve physics, number, space etc.; see e.g., Hauser and Spelke 2004; Santos et al. 2001; Shutts et al. 2009; Spelke and Kinzler 2007). The accounts range from strong domain-specific nativism (there are innate domain-specific beliefs-for example, in the domain of food that color is a reliable indicator of identity, whereas in the domain of tools, color is irrelevant but shape matters.) via intermediate positions (e.g., there are domain-specific learning mechanisms that gradually lead to differential sensitivity to different properties in different domains) to purely domain-general accounts (e.g., that there is only one kind of general purpose learning mechanism that inductively picks up on different diagnostic values of different properties in different domains; for an excellent exposition of this logical space of possible accounts, see Shutts et al. 2009). The fact that some experience-in fact, very little experience-can make properties available for food object individuation that are not spontaneously used (i.e., shape), speaks against any strong domain-specific nativist position. In this respect, the present findings are in line with developmental data that differential attention to color as diagnostic property for food individuation and induction is a relatively late developing phenomenon that only arises after infancy (Shutts et al. 2009). Both of these lines of research taken together thus narrow down the logical space of accounts to such construals that either posit weaker domain-specific learning mechanisms (rather than strongly innate domainspecific beliefs) or that posit domain-general learning mechanisms leading to domain-specific predispositions (e.g., Karmiloff-Smith 1992). Needless to say, more comparative and developmental research is needed to decide between these and further narrow down the hypothesis space.

Second, the present findings have some implications regarding object individuation in non-human animals. Against the background of the debate about potentially uniquely human and linguistically constituted property/ kind-based object individuation, the present findings corroborate previous findings that property-based object individuation is possible in the absence of language. Moreover, the findings suggest that property/kind-based object individuation in primates is not just a very limited phenomenon in some very restricted domain, say for just one kind of property, but seems to be a more general and reliable ability extending to different types of properties. What the present findings do not tell us, however, is what underlies primates' competence in the kinds of tasks used here. In particular, do these tasks tap true kind-based object individuation, or do they just measure sophisticated tracking of features (see Mendes et al. 2008; Xu 2002). What we need in future research to decide between these different possibilities are tasks that tease apart property and kind information. This could be done, for example, by introducing different kinds of object transformations (property transformations pitted against kind transformations; see, e.g., Feigenson and Carey 2003; Xu et al. 2004, 
for some attempts in these directions in infancy work). The use of match-to-sample tasks (based on color and shape properties) might also help to clarify the aforementioned question.

Acknowledgments This research was partially supported by a Ph.D. grant from the "Fundação para a Ciência e a Tecnologia", Portugal to Natacha Mendes. We are grateful to Antje Girndt, Heinz Gretscher, and Johannes Grossman for assistance with the data collection, and Corinna Zrout for reliability coding. Thank you to Raik Pieszek for the construction of the experimental apparatus. Thanks also to the zookeepers at the Leipzig Zoo for their help with the apes.

Open Access This article is distributed under the terms of the Creative Commons Attribution Noncommercial License which permits any noncommercial use, distribution, and reproduction in any medium, provided the original author(s) and source are credited.

\section{References}

Bania AE, Harris S, Kinsley HR, Boysen ST (2009) Constructive and deconstructive tool modification by chimpanzees (Pan troglodytes). Anim Cogn 12:85-95. doi:10.1007/s10071-008-0173-0

Feigenson L, Carey S (2003) Tracking individuals via object-files: Evidence from infants' manual search. Dev Sci 6:568-584. doi: 10.1111/1467-7687.00313

Furlong EEMJ, Boose KJ, Boysen ST (2008) Raking it in: the impact of enculturation on chimpanzee tool use. Anim Cogn 11:83-97. doi:10.1007/s10071-007-0091-6

Haccou P, Meelis E (1994) Statistical analyses of behavioural data. Oxford University Press, Oxford

Hauser MD, Spelke ES (2004) Evolutionary and developmental foundations of human knowledge: a case study of mathematics. In: Gazzaniga M (ed) The cognitive neurosciences, vol 3. MIT Press, Cambridge

Hauser MD, Pearson HM, Seelig D (2002) Ontogeny of tool-use in cotton-top tamarins (Saguinus oedipus): innate recognition of functionally relevant features. Anim Behav 64:299-311. doi: 10.1006/anbe.2002.3068

Karmiloff-Smith A (1992) Beyond modularity: a developmental perspective on cognitive science. MIT Press, Cambridge

Mendes N, Rakoczy H, Call J (2008) Ape metaphysics: object individuation without language. Cognition 106:730-749. doi: 10.1016/j.cognition.2007.04.007
Phillips W, Santos L (2007) Evidence of kind representations in the absence of language: experiments with rhesus monkeys (Macaca mulatta). Cognition 102:455-463. doi:10.1016/j.cognition.2006. 01.009

Santos LR, Hauser MD, Spelke ES (2001) Recognition and categorization of biologically significant objects by rhesus monkeys (Macaca mulatta): the domain of food. Cognition 82:127-155. doi:10.1016/S0010-0277(01)00149-4

Santos LR, Sulkowski GM, Spaepen GM, Hauser MD (2002) Object individuation using property/kind information in rhesus macaques (Macaca mulatta). Cognition 83:241-264. doi:10.1016/ S0010-0277(02)00006-9

Santos LR, Miller CT, Hauser MD (2003) Representing tools: how two non-human primate species distinguish between the functionally relevant and irrelevant features of a tool. Anim Cogn 6:269-281. doi:10.1007/s10071-003-0171-1

Santos LR, Pearson HM, Spaepen GM, Tsao F, Hauser MD (2006) Probing the limits of tool competence: experiments with two non-tool-using species (Cercopithecus aethiops and Saguinus oedipus). Anim Cogn 9:94-109. doi:10.1007/s10071-005-0001-8

Shutts K, Condry KF, Santos LR, Spelke ES (2009) Core knowledge and its limits: the domain of food. Cognition 112:120-140. doi: 10.1016/j.cognition.2009.03.005

Spelke ES, Kinzler KD (2007) Core knowledge. Dev Sci 10:89-96. doi:10.1111/j.1467-7687.2007.00569.x

Uller C, Carey S, Hauser MD, Xu F (1997) Is language needed for constructing sortal concepts? A study with non-human primates. In: Hughes E, Hughes M, Greenhill A (eds) Proceedings of the 21st annual Boston university conference on language development. Cascadilla Press, Sommerville, pp 665-677

$\mathrm{Xu} \mathrm{F}$ (2002) The role of language in acquiring object kind concepts in infancy. Cognition 85:223-250. doi:10.1016/S0010-0277(02) 00109-9

Xu F (2007) Sortal concepts, object individuation, and language. Trends Cogn Sci 11:400-406. doi:10.1016/j.tics.2007.08.002

Xu F, Carey S (1996) Infants' metaphysics: the case of numerical identity. Cogn Psychol 30:111-153. doi:10.1006/cogp.1996. 0005

Xu F, Carey S, Quint N (2004) The emergence of kind-based object individuation in infancy. Cogn Psychol 49:155-190. doi: 10.1016/j.cogpsych.2004.01.001

Xu F, Cote M, Baker A (2005) Labeling guides object individuation in 12-months-old infants. Psychol Sci 16:372-377. doi:10.1111/ j.0956-7976.2005.01543.x 